\title{
Impactos de las actividades antrópicas en el nevado Huaytapallana
}

\author{
Impacts of anthropic activities on the nevado Huaytapallana
}

Jacinto Arroyo Aliaga', Natalie Schulz ${ }^{2}$, Pedro Gurmendi Párraga ${ }^{3}$

Instituto Geofísico del Perú

\section{RESUMEN}

Objetivos: Explicar la influencia de las actividades antrópicas en la variación de las capas de acumulación de nieve en los glaciares de la vertiente sur-occidental del nevado Huaytapallana de los Andes Centrales del Perú, en el ciclo hidrológico 2011-2012. Métodos: Se utilizó el método general hipotético - deductivo como guía en la investigación cuantitativa; el método del Thornthwaite para realizar el análisis hidrológico y clasificación climática de la zona; y el balance de masas como método para estimar la carga o descarga de nieve acumulado mediante balizas. Resultados: Se ha encontrado que las actividades antrópicas de turismo vivencial es una de las actividades principales que afectan al glaciar con el $60 \%$ de impacto. Las actividades de reforestación constituyen el $20 \%$ de impacto y el $10 \%$ la actividad de pastoreo. También los impactos de las actividades antrópicas a altitudes bajas en el glaciar son negativas, mientras que a altitudes superiores a los $5000 \mathrm{msnm}$ se registran menores impactos negativos. De igual manera se encontró una relación directa (coeficiente $r=0,97$ ) entre el impacto a bajas altitudes de las masas glaciares. Conclusiones: La actividad de turismo vivencial que se realiza en varios sectores del nevado Huaytapallana, es una de las principales actividades antrópicas que está generando impactos negativos en el sistema glaciar al acelerar la pérdida de masa de nieve acumulado durante un ciclo hidrológico.

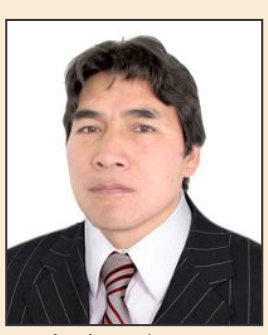

Jacinto Arroyo

Palabras clave: Impactos glaciares, actividades antrópicas, nevado Huaytapallana

1 Investigador adjunto en prevención de desastres y cambio climático del Instituto Geofísico del Perú.

2 Docente investigadora de la Pontificia Universidad Católica del Perú.

3 Docente de la Universidad Continental. 


\section{ABSTRACT}

Objectives: To explain the influence of the anthropic activities on the variation of the snow accumulation layers on glaciers in the south-west drainage basin of nevado Huaytapallana of the central Andes of Peru in the hydrological cycle 2011-2012. Methods: We used the general method hypothetical - deductive to guide the quantitative research, the Thornthwaite method to perform the hydrological analysis and the climate classification of the area, in addition the mass balance like the method for estimating the bulk and volley of accumulated snow by beacons. Results: We found that anthropic activities of experiential tourism are one of the main activities that affect the glacier with $60 \%$ of impact. Reforestation activities constitute $20 \%$ of impact and $10 \%$ the activity grazing. Also the impact of anthropic activities at low altitudes in the glacier are negative, while at latitude above $5000 \mathrm{msl}$ are recorded minor negative impact. Similarly we found a direct relationship (coefficient $r=0,97$ ) between the impact at low altitudes of glacier masses. Conclusions: The experiential tourism activity that takes place in various sectors of the nevado Huaytapallana, is one of the main anthropic activities that is generating negative impacts in the glacial system becouse it accelerate the mass loss of accumulated snow during a hydrological cycle.

Keywords: Glacier impact, anthropic activities, nevado Huaytapallana.

\section{INTRODUCCIÓN}

Se tiene pruebas basadas en registros de observaciones glaciológicas que en los últimos años diferentes actividades antrópicas vienen alterando el sistema natural de acumulación y pérdida de masa de los glaciares. Estas actividades hacen que directamente las zonas de ablación se vuelvan más vulnerables.

Además de las causas directas por las actividades industriales y comerciales de los seres humanos que ha permitido que las concentraciones de gases de efecto invernadero, en particular el dióxido de carbono aumente en un 35\%, valores superiores comparados a otros años y que están causando impactos negativos a los glaciares (1). Se suman las actividades mecánicas de acción directa en los mismos glaciares.

Las actividades antrópicas directas están contribuyendo a acelerar la pérdida del manto de nieve natural que cubre a los glaciares y que les protege de acumulación neta de energía. Y de continuar con tales actividades los glaciares se verán acelerados en su desglaciación.

Las preguntas planteadas son:ટ̇de qué manera las actividades antrópicas influyen en la variación de las capas de acumulación de nieve en los glaciares de la vertiente suroccidental del nevado del Huaytapallana en el Perú?, żcuáles son las características de las actividades antrópicas que causan impactos en el sistema glaciológico?, ¿̇cuáles son las características climáticas e hidrológicas del sistema glaciológico del nevado? ¿̇qué relación existe entre los tipos de actividades antrópicas y la variación de las capas de acumulación de nieve?

Los objetivos intentaron explicar los impactos directos de la acción antrópica en el sistema de acumulación de masa glaciar, identificando y caracterizando los tipos de actividades que causan impactos. Además, se determinaron las relaciones entre estas actividades y su influencia en la aceleración de tales pérdidas.

Los glaciares del nevado Huayłapallana, constituyen por el momento una gran reserva hídrica para la ciudad de Huancayo.

Entre las diferentes actividades antrópicas que ejercen una influencia directa en los sistemas glaciares, el turismo vivencial que se practica, constituye el principal motor de 
Tabla N 01: Localización geográfica de los puntos de evaluación.

\begin{tabular}{llll}
\hline Puntos & Latitud & Longitud & Altitud \\
\hline Baliza 1 & $8681049,66 \mathrm{~S}$ & $494964,06 \mathrm{E}$ & $4917 \mathrm{msnm}$ \\
Baliza 2 & $8681247,77 \mathrm{~S}$ & $494939,72 \mathrm{E}$ & $4980 \mathrm{msnm}$ \\
Baliza 3 & $8681523,02 \mathrm{~S}$ & $494730,00 \mathrm{E}$ & $5050 \mathrm{msnm}$ \\
Baliza 4 & $8681522,19 \mathrm{~S}$ & $494339,68 \mathrm{E}$ & $5080 \mathrm{msnm}$ \\
Baliza 5 & $8681842,23 \mathrm{~S}$ & $494632,11 \mathrm{E}$ & $5200 \mathrm{msnm}$ \\
\hline
\end{tabular}

impactos directos al sistema de pérdida de masa glaciar en las zonas de ablación y de borde. Porque esta actividad hace que se acelere la pérdida de masa acumulada de nieve que se tuvo durante los ciclos hidrológicos.

Las actividades antrópicas influyen sobre las capas de acumulación de masa glaciar de la vertiente sur-occidental del nevado Huaytapallana al impactar significativamente en la variación del sistema de acumulación por acción directa del turismo vivencial y por acciones indirectas de las actividades de reforestación, pastoreo, zanjas de infiltración y actividades culturales de la población, elevando la isoterma a niveles más altos cercanos a los glaciares durante el ciclo hidrológico 2011-2012.

\section{MATERIAL Y MÉTODOS}

El alcance del estudio fue de carácter cuantitativo, las variables de estudio fueron clasificados como variables discretas para los datos de fenómenos antropogénicos y continuas para los datos climáticos y de evaluación del glaciar. Además, se clasificaron las variables por su dominio en dependientes e independientes. Se utilizaron los métodos teóricos o generales para

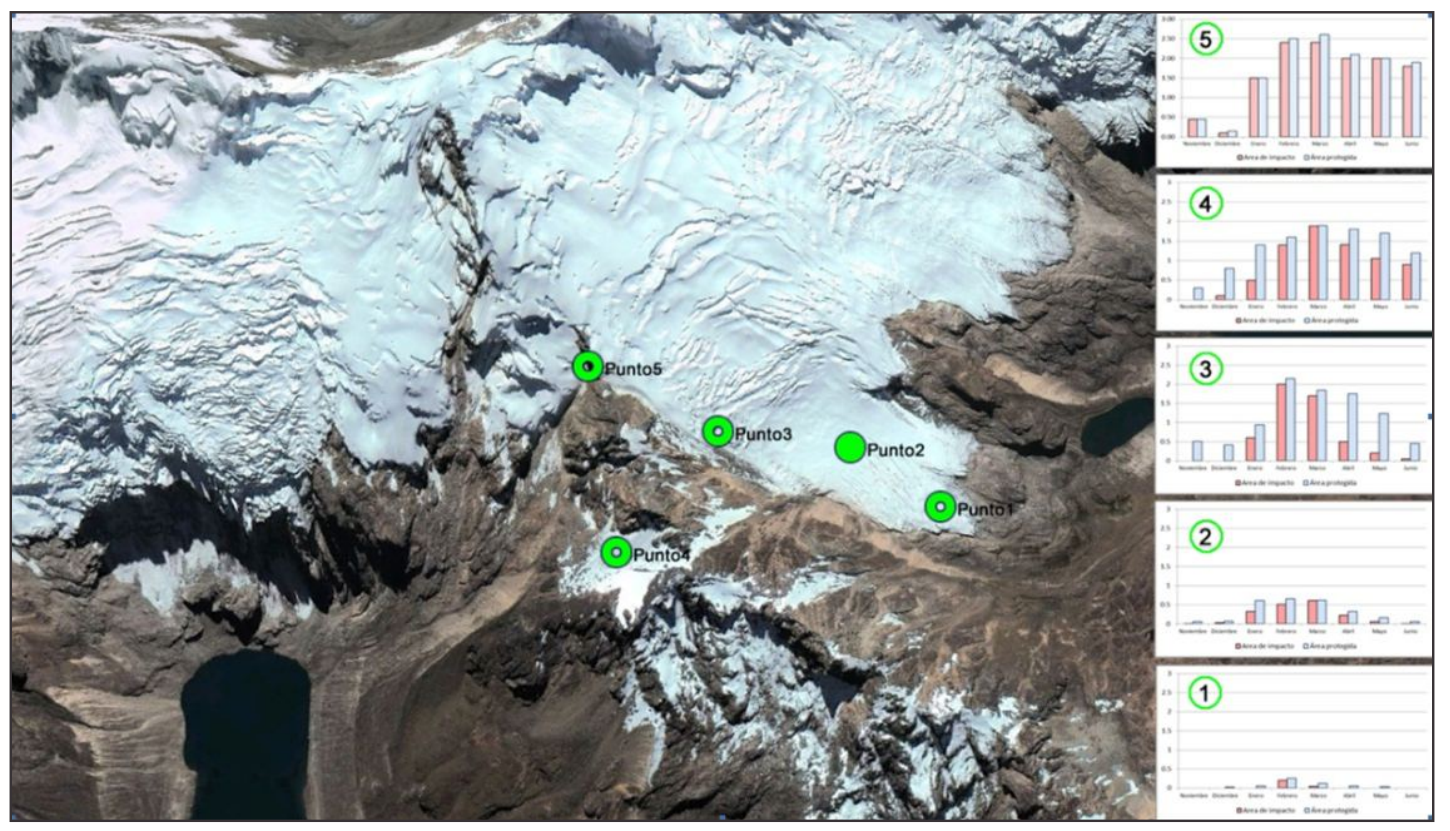

Figura 01: Zonas de evaluación de impacto antrópico al glaciar por actividades realizadas. 
direccionar la investigación y los métodos específicos para entender la aplicación real del problema.

Se utilizó el método general hipotéticodeductivo para recolectar la información de base. Este método combina la reflexión racional o momento racional de las relaciones entre los glaciares y las actividades antrópicas con la observación de la realidad o momento empírico. También, se utilizó el método teórico analítico para descomponer los fenómenos antrópicos de turismo vivencial, pastoreo, zanjas de infiltración, reforestación y actividades culturales en sus elementos constitutivos. Este método favorece la descomposición de cada variable en partes que pueden estudiarse para demostrar que las conclusiones son una consecuencia de las premisas a partir de los conocimientos.

Los métodos utilizados fueron, de registro de campo y de trabajo en gabinete. En el trabajo de campo se han trabajado en diferentes pases secuenciales, empezando con la fase exploratoria en diferentes puntos del nevado Huaytapallana, para luego instalar balizas en puntos geo referenciados, en la fase de monitoreo se han obtenido registros in situ para su respectivo análisis. En la etapa de gabinete se han elaborado mapas temáticos a partir de imágenes satelitales.

\section{Puntos de Evaluación}

La figura 01, muestra una imagen del lugar en dónde se instalaron las balizas y se tomaron las muestras en dos condiciones, que consiste en instalar balizas en zonas con intervención y otro en zonas sin intervención. Por lo agreste de la zona de estudio sólo se instalaron cinco balizas; figura 02 .

\section{Estimación del balance glaciológico}

Para estimar el balance glaciológico, se utilizó la ecuación básica del balance (b) de masa en un punto del glaciar para un instante, $\mathrm{db} / \mathrm{dt}$, que describe muy bien Lliboutry, Paterson y Dyurgerov. $\frac{\mathrm{db}}{\mathrm{dt}}=\rho \frac{\mathrm{dh}}{\mathrm{dt}}+\int \frac{\mathrm{d} \rho}{\mathrm{dt}} \mathrm{dy}$

Donde $\rho$ es la densidad del hielo de espesor h que varía según el tiempo t. El primer término del lado derecho de la ecuación representa el cambio de masa de hielo (con densidad constante). El segundo término es el cambio de densidad de la columna de nieve de espesor y sobre el periodo de tiempo t (figura 01).

A partir de (a) y considerando dos instantes de medición, ti y ti-1, se obtiene el balance de masa para el instante i como,

Considerando $\rho_{\mathrm{i}}=\rho_{\mathrm{i}-1}=\rho$ se tiene:

$\mathrm{b}_{\mathrm{i}}=\rho_{\mathrm{o}} \Delta \mathrm{h}+\left(\rho y_{\mathrm{i}}-\rho_{\mathrm{i}-1} y_{\mathrm{i}-1}\right)$

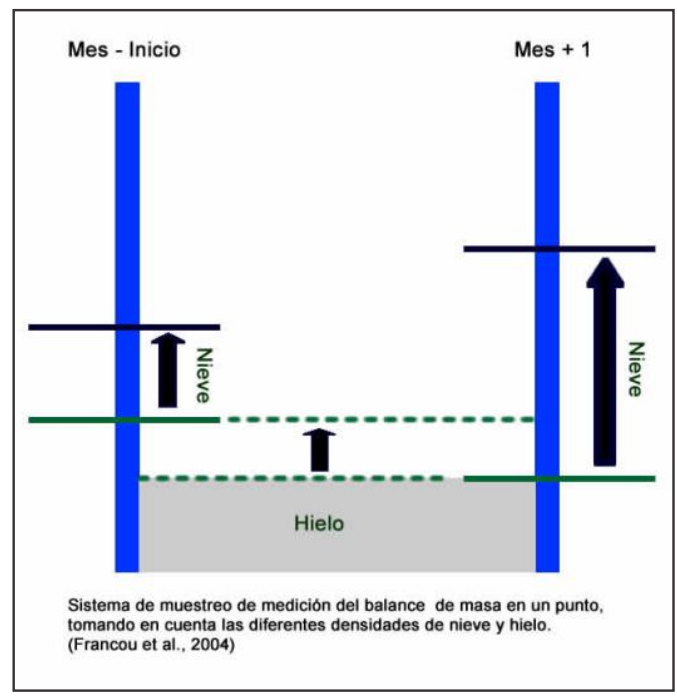

Figura 02: Diagrama del estado de una baliza para dos instantes consecutivos. Aquí puede observarse la altura de emergencia (z), nieve (y) y hielo (h) para los instantes respectivos.

$\mathrm{b}_{\mathrm{i}}=\rho_{\mathrm{O}}\left(\mathrm{h}_{\mathrm{i}}-\mathrm{h}_{\mathrm{i}-1}\right)+\rho\left(y_{\mathrm{i}}-y_{\mathrm{i}-1}\right)$

A escala anual, para extender el balance a todo el glaciar se utilizan diversos puntos de medición (balizas y pozos) distribuidos en una red bien definida. El principio básico es el de ponderar el balance medido por el área del rango relativo del glaciar.

El procesamiento de datos se lleva a cabo a través de tres etapas. La primera 
corresponde a la división del glaciar en rangos de altura que fluctúa generalmente entre 50 y 100 m (aunque en glaciares pequeños, los rangos pueden ser de $25 \mathrm{~m}$ ). En la segunda etapa, se calculan los valores medidos para cada rango. En el caso de que ninguna baliza haya sido instalada en un rango, los valores del balance son obtenidos por interpolación. Finalmente, el balance neto específico $\mathrm{Bn}$, es el valor de balance ponderado por su superficie relativa utilizando la siguiente ecuación:

\section{$\mathrm{Bn}=\Sigma \mathrm{Bi}(\mathrm{si} / \mathrm{S})$}

Donde $\mathrm{Bi}$ corresponde al balance de un rango de altura i, si la superficie del rango de altura y $\mathrm{S}$ a la superficie total del glaciar.

\section{Escurrimiento a partir de la fusión de nieve y de hielo}

El modulo glaciar se basa en una adaptación de modelo Grado-Día propuesto por Hock (4) para que trabaje al paso del tiempo mensual. Otros autores utilizaron este tipo de modelo en los Andes, Suarez, et al (6) y en los Alpes, Schaeffli, et al. (7).

El escurrimiento desde el glaciar sigue las ecuaciones a y b.

$$
\begin{aligned}
& Q_{\text {hielo }}=\left[a_{\text {hielo }}\left(T_{(\mathrm{ti})}-\mathrm{T}_{\mathrm{o}}\right)\right]_{\text {si }} \mathrm{T}_{(\mathrm{ti})} \geq \mathrm{T}_{\mathrm{o}} \\
& \mathrm{Q}_{\text {hielo }}=0 \text { si } \mathrm{T}_{(\mathrm{ti})}<\mathrm{T}_{\mathrm{o}}
\end{aligned}
$$

Donde, $Q_{\text {hielo }}(\mathrm{mm})$ viene a ser el escurrimiento producido por fusión del hielo en el tiempo (ti), $\mathrm{T}\left({ }^{\circ} \mathrm{C}\right)$ es la temperatura del aire, To $\left({ }^{\circ} \mathrm{C}\right)$ la temperatura límite y a $\left(\mathrm{mm} / \mathrm{mes}^{*}\right.$ ${ }^{\circ} \mathrm{C}$ ) es el coeficiente de fusión. De estos, la temperatura To y el a son dos parámetros de calibración del módulo glaciar y la temperatura T, la variable de activación.

En cuanto a la nieve, el modelo permite por cada banda producir una capa en evolución (acumulación y derretimiento). Se acumula nieve si la precipitación ocurre bajo una temperatura más baja que la temperatura límite y se produce una fusión de la capa de nieve cuando la temperatura del aire sobrepasa la temperatura límite.

Para el caso de la fusión de nieve se mantiene la misma ecuación cambiando el sufijo hielo por nieve y también los parámetros de nieve. Finalmente, solo 3 parámetros controlan la acumulación y la fusión de la nieve y del hielo (To, ahielo, anieve).

\section{Dinámica de acumulación y pérdida de masa glaciar}

Para simular la evolución de los glaciares en términos de volumen y de área, se calcula cada mes y por cada banda (o catchment) glaciar el balance de masa (diferencia entre la acumulación y la ablación). Después, a la escala anual se calcula si el glaciar acumuló o perdió hielo. Para estimar el volumen de hielo en el glaciar, el modelo se basa sobre la relación propuesta por Bahr, et al. (8)

$\mathrm{V}_{\text {glaciar }}=\mathrm{c} * \mathrm{~A}_{\text {glaciar }}^{\mathrm{b}}$

Donde, $V(m 3)$ es el volumen glaciar, $A(m 2)$ es el área glaciar calculada sobre la base de imágenes de satélite, $b=1,36$ y c $=0,048$ son constantes calculadas en función a observaciones hechas en 144 glaciares del mundo. Con el conocimiento de la densidad del hielo $(0,9 \mathrm{gr} / \mathrm{cm} 3)$ podemos calcular el área de la pérdida o ganancia de masa glaciar. Para una descripción detallada del funcionamiento de la parte glaciar del modelo, se recomienda reportarse al artículo Condom, et al. (9).

\section{RESULTADOS}

La figura 03, muestra las principales actividades antrópicas detectadas, donde el turismo vivencial tiene el $60 \%$ de impactos sobre el glaciar en los sistemas de borde. La segunda actividad que está alterando un calentamiento repentino al sistema glaciar es la reforestación.

La figura 04, muestra la ubicación geográfi- 


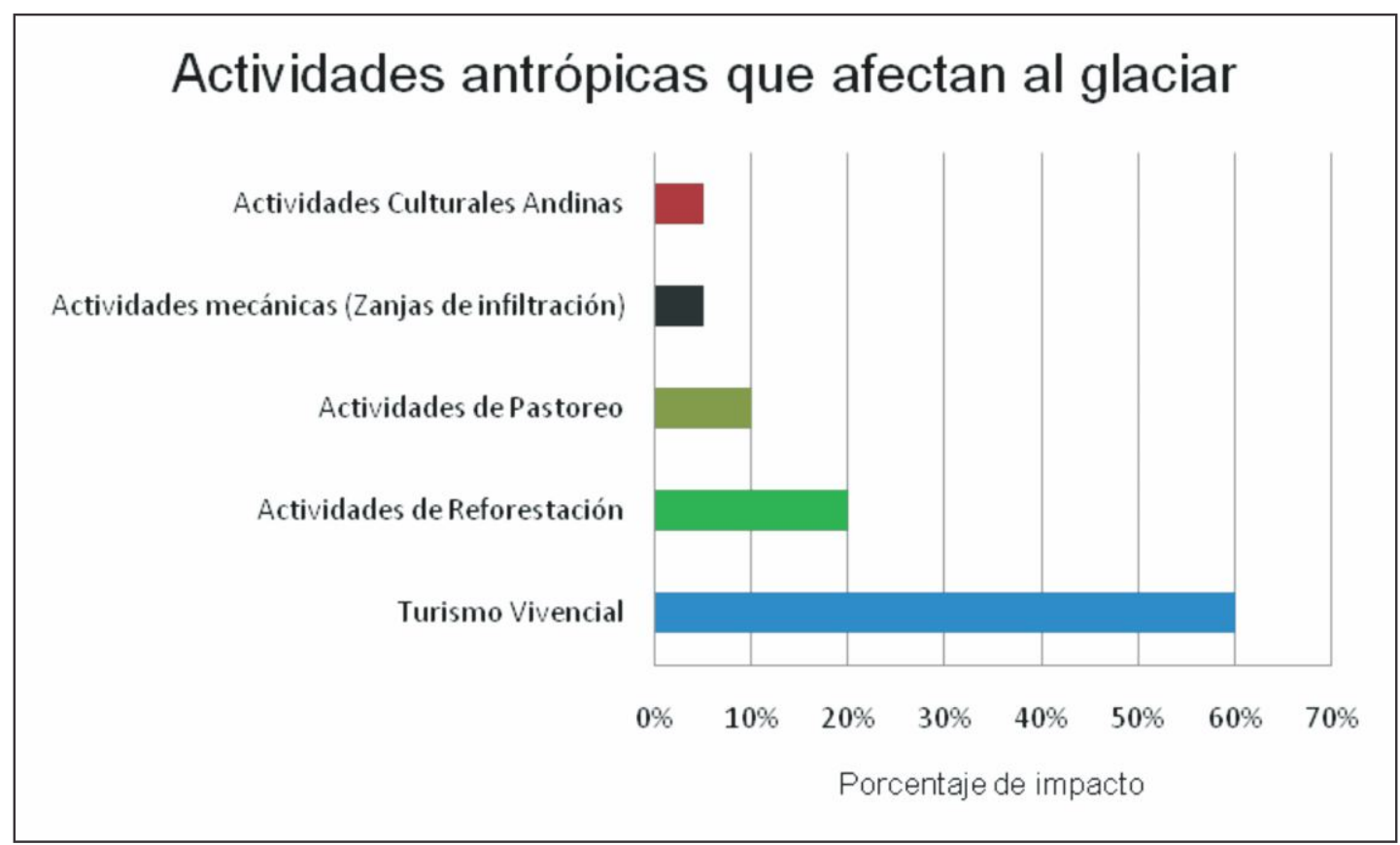

Figura 03: Actividades antrópicas detectadas en el nevado Huaytapallana.

ca del nevado Huaytapallana. Se caracteriza las zonas de impacto por las diferentes actividades antrópicas que se realizan en el glaciar. Los deslizamientos originados por el retroceso glaciar son comunes en los bordes occidentales de los glaciares cercanos a las lagunas. El pastoreo es una actividad que está incidiendo en los glaciares, acelerando los nevados acumulados. Las zanjas de infiltración, son otras actividades que están alterando las zonas de ablación.

La figura 05, muestra las actividades antrópicas de turismo vivencial, que daña las capas de los glaciares. Las actividades son críticas en los meses del invierno y primavera, porque afecta al mismo glaciar que tiene una coloración diferente a las nieves y retiene calor energético, acelerando la pérdida de masa glaciar.

Diferentes observaciones realizadas en los glaciares del nevado, para evaluar los impactos que causan las actividades antrópicas, especialmente las diversas actividades turísticas vivenciales que se realiza sobre el glaciar, ha proporcionado información sobre el daño que está causando estas actividades sobre la capa de nieve granular que recubre y protege a los glaciares (figura 06).

En la zona de ablación, donde el glaciar debería perderse naturalmente por procesos físicos y mecánicos que ocurre en el ciclo natural del glaciar, la intervención humana mediante sus actividades ésta impactando a los nevados de la siguiente manera. Las huellas de zapatos que se dejan al subir o caminar en el nevado del glaciar (figura $07 \mathrm{y}$ 08), están dejando vulnerable el hielo que tiene una coloración distinta a la de la nieve, de color marrón oscuro o negro que corresponde a diferentes gases como el $\mathrm{CO} 2, \mathrm{CH} 4, \mathrm{NO} 2$ y diferentes partículas que han sido atrapados en el proceso de solidificación del glaciar.

El impacto al glaciar también se realiza por la inmensa remoción de material de nieve granular que se realiza en la zona conocida como lengua del glaciar. Los deslizamientos que realizan los turistas desde las partes cercanas a las zonas de acumulación como parte de su diversión están dejando áreas descubiertas que permiten un recalentamiento acelerado del hielo creando un proceso de sublimación y fusión del glaciar (figura 09). 


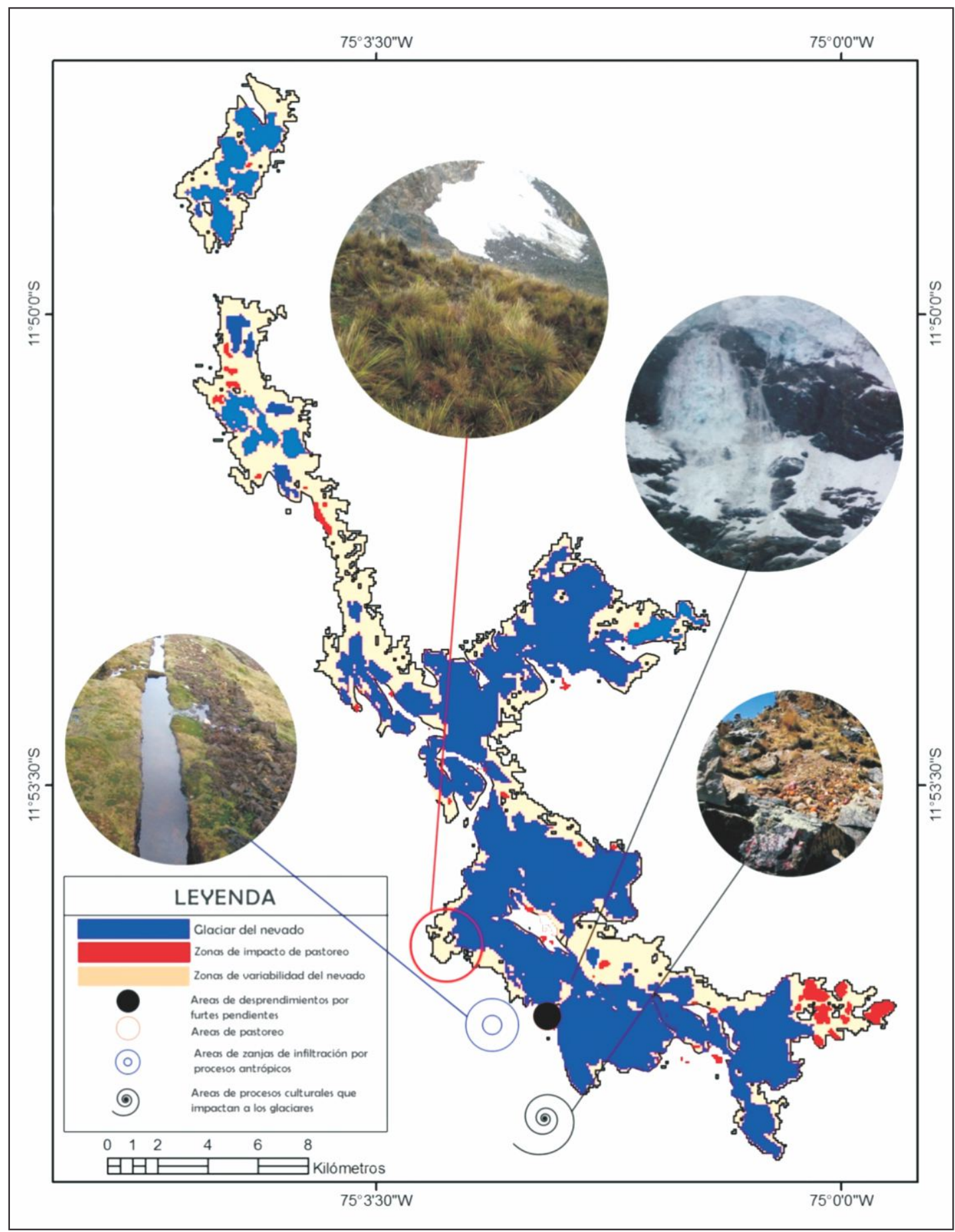

Figura 04: Representación gráfica de los puntos donde se concentran las actividades antrópicas.

La zona de ablación del glaciar muestra una pérdida acelerada de masa glaciar cuando la intervención humana es directa, como se muestra en la figura 10, donde la zona de ablación ha perdido toda la capa de nieve que lo protege de la fusión y la vulnerabilidad está acelerando la desglaciación por los efectos antrópicos.

La climatología de la zona de estudio (figura 11) corresponde según la clasificación de Köppen a un clima de montaña glaciar, con 


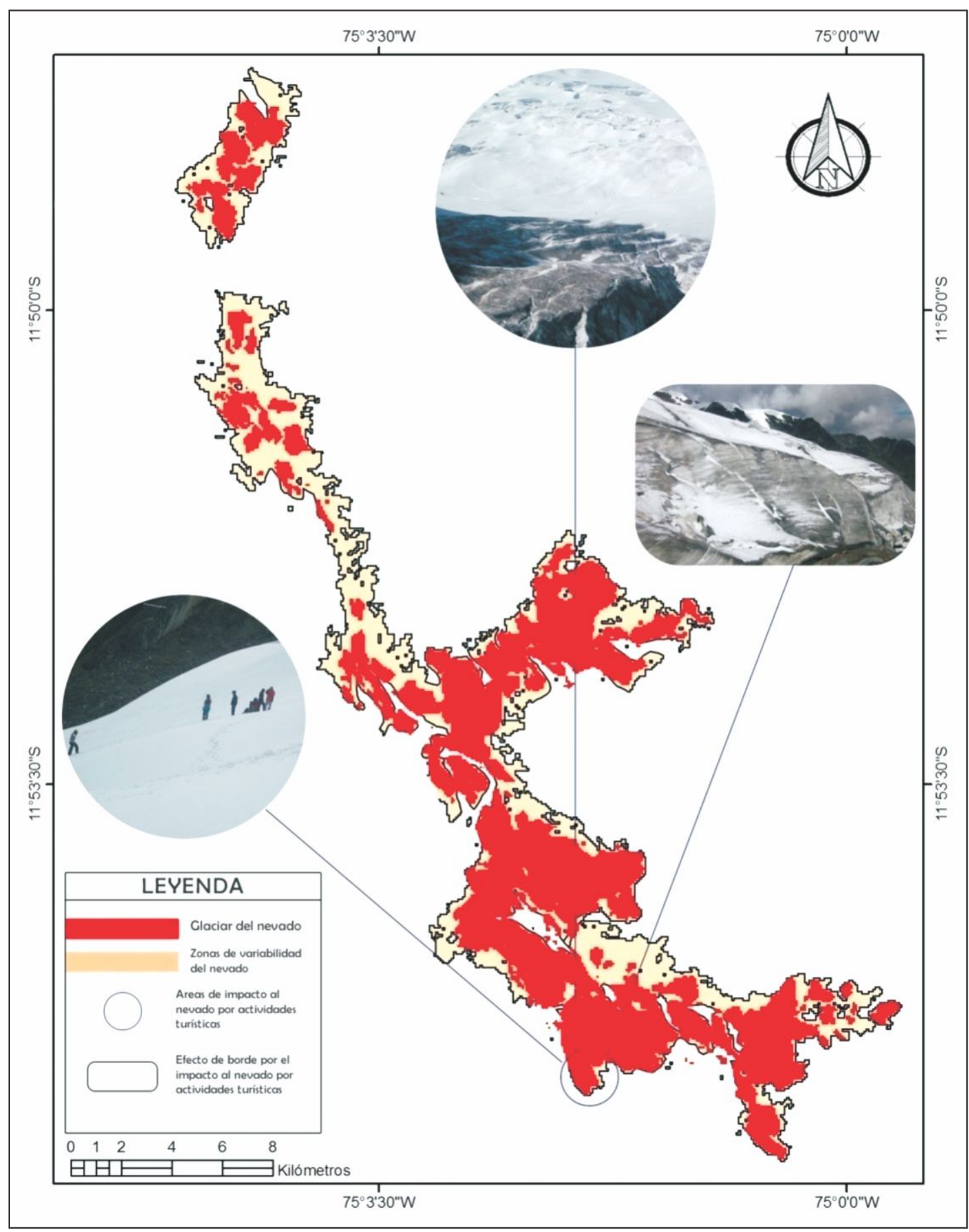

Figura 05: Zonas de impacto de actividades de turismo vivencial

temperatura promedio mensual inferior a $0^{\circ} \mathrm{C}$ durante el año y temperatura máxima de $12^{\circ} \mathrm{C}$. Los meses que corresponden al verano hay un ligero aumento de la temperatura por su estacionalidad, subiendo la temperatura mínima en $2^{\circ} \mathrm{C}$. Las precipitaciones también tienen a estar regulados por un ciclo estacional que corresponde a los meses de noviembre a marzo.

La figura 12, muestra el resultado 


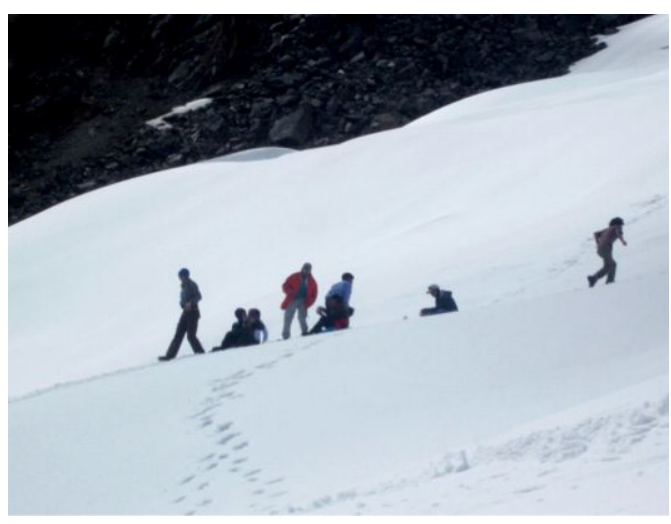

Figura 06: Actividad turística en el nevado Huaytapallana

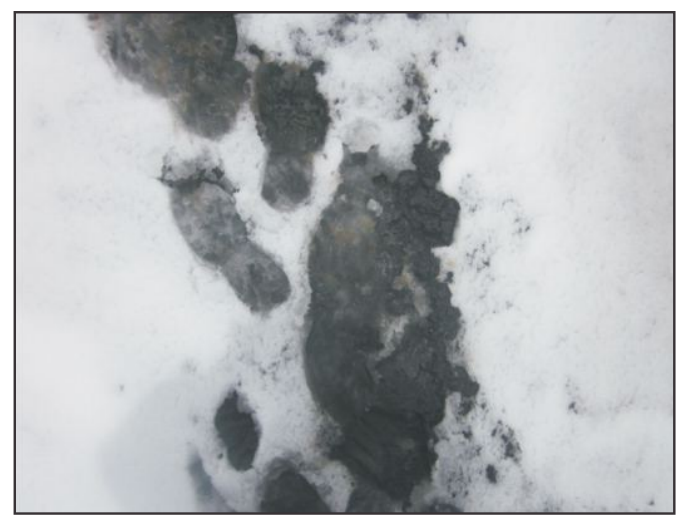

Figura 07: Pérdida de estratos superficiales de nieve por actividades turísticas.

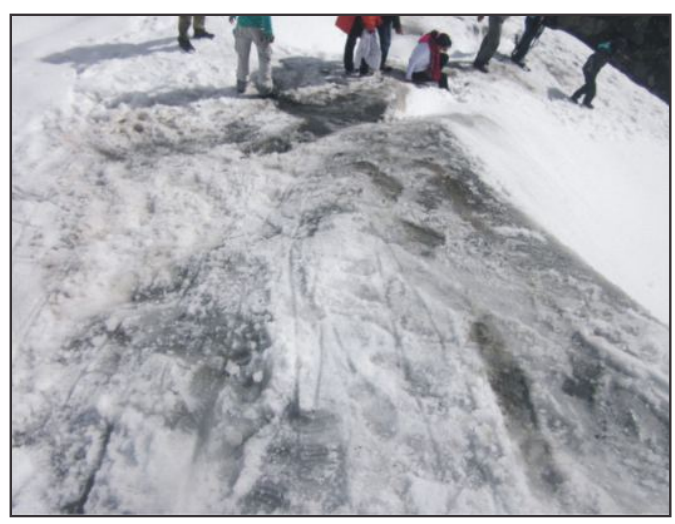

Figura 08: Impactos en la nieve granular y neviza.

acumulado de la pérdida de masa glaciar de todos los puntos con respecto a las zonas no impactadas. Las áreas de impacto en donde las pérdidas de masa glaciar son significativas empieza a partir del punto de

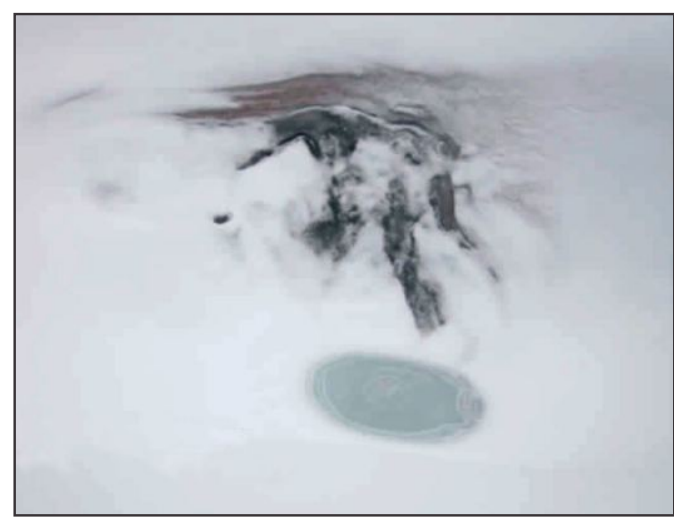

Figura 09: Formación de lagunas en el nevado.

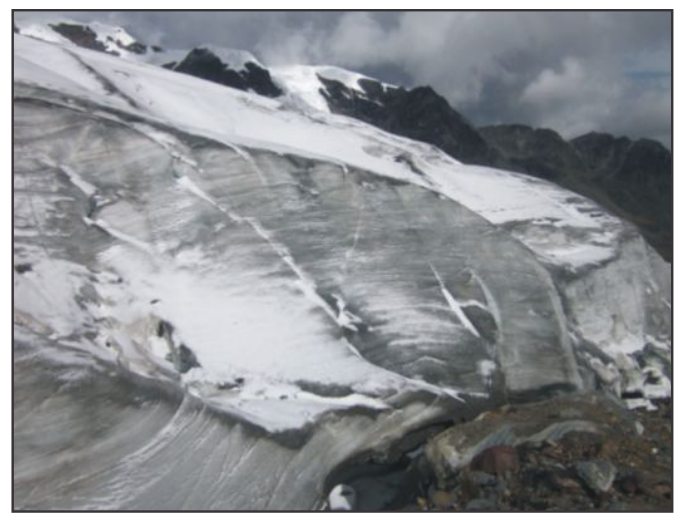

Figura 10: Zonas de ablación totalmente desprotegidas de capa de nieve granular

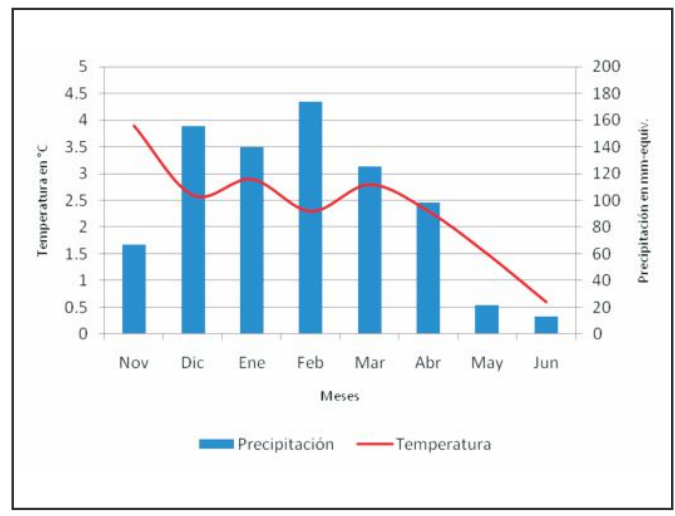

Figura 11: Distribución mensual de la temperatura y la precipitación.

observación uno y se extiende hasta el punto cuatro. El punto cinco es el único punto en dónde la acumulación y la pérdida por impacto es imperceptible. 


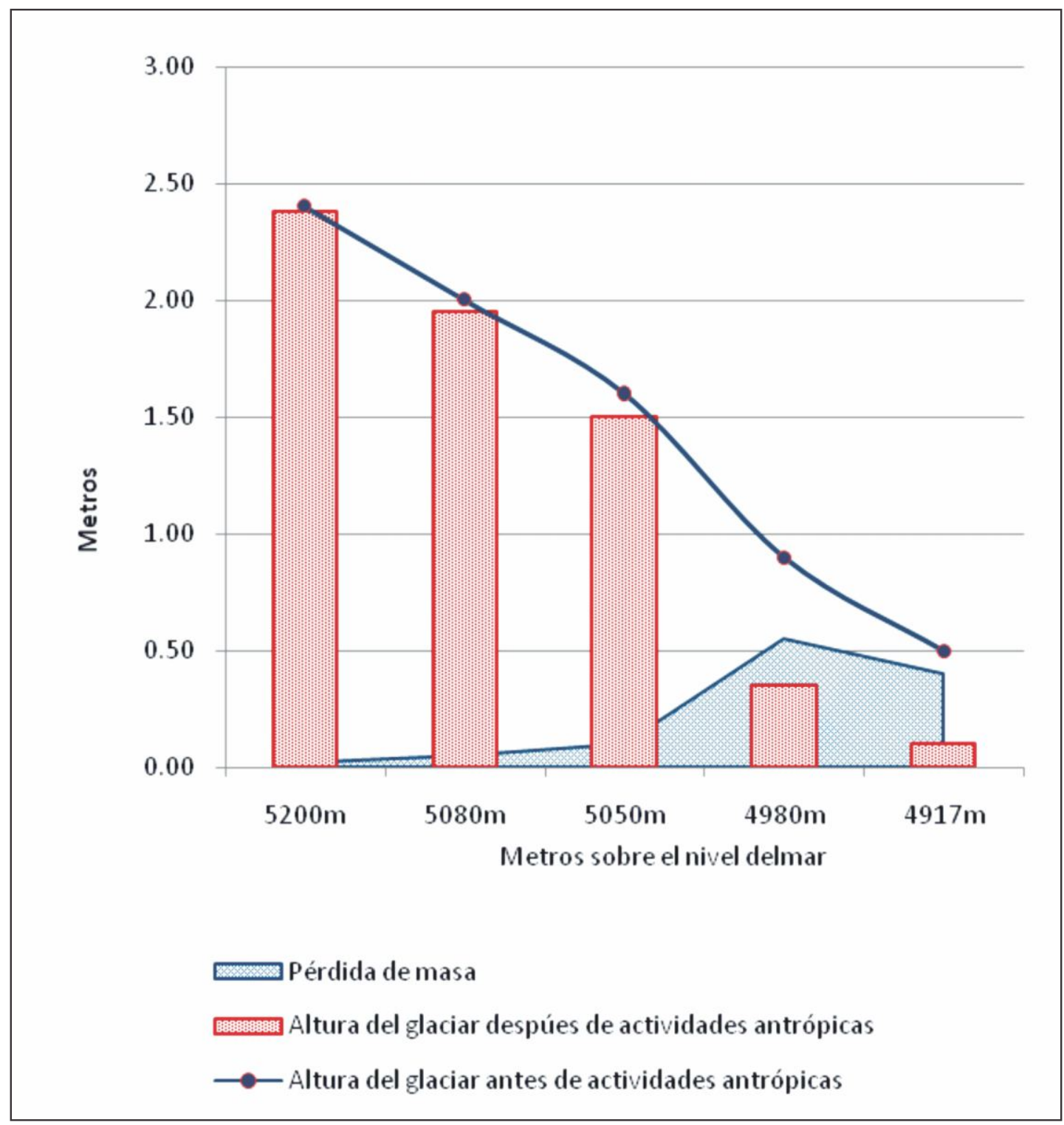

Figura 12: Diferencias totales de acumulación entre zonas de impacto y zonas de muestreo.

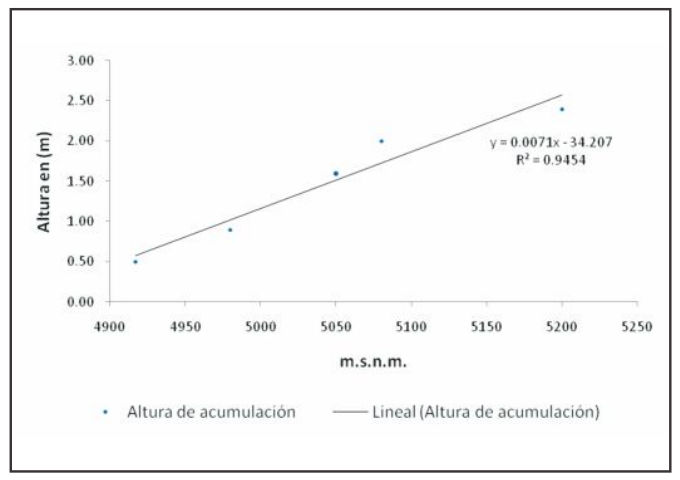

La figura 13, muestra la correlación entre la altura de conservación por actividades del turismo vivencial y la altitud sobre el nivel del mar en dónde son frecuentes las actividades. La pendiente de conservación $\frac{\Delta y}{\Delta x}=0,007$ es mayor con la altitud. El coeficiente de correlación $r=0,97$.

Figura 13: Relación de pérdida de nieve acumulada en función de la altitud. 


\section{DISCUSIÓN}

La actividad antrópica que más impacta sobre las capas de acumulación de masa glaciar de la vertiente sur-occidental del nevado Huaytapallana, es el turismo vivencial, al alterar la variación del sistema de acumulación por acciones directas. Las actividades de reforestación, pastoreo, zanjas de infiltración y actividades culturales de la población tienen un impacto indirecto en el nevado Huaytapallana (figura 03).

Las actividades de turismo vivencial afectan directamente el proceso de acumulación de las zonas bajas o zona de ablación (figura 11), al estar expuesto por su altitud (4 917 msnm) a una continua actividad turística. Dicho proceso deja expuesto extensas zonas de hielo oscuro (figura 07 y 08). La conservación de la primera capa de nieve es importante para el glaciar, porque el color blanquecino que recubre al glaciar, permite procesos físicos que mantienen el equilibrio térmico, haciendo que la radiación incidente se pierda inmediatamente por procesos del fenómeno del albedo y evitar procesos de acumulación de energía.

La nieve debería seguir el proceso natural de diagénesis, que consiste en el proceso de transformación de la nieve depositada en hielo glaciar. Este proceso es una condición básica y necesaria que depende esencialmente de la presión y temperatura a partir de la conservación del estrato de nieve fresca, para luego seguir con la compactación temporal, hasta la formación de hielo glaciar. Este proceso debería ocurrir desde el primer momento de la tormenta de nieve, hasta culminar el ciclo, entendiendo que debe registrarse mayores precipitaciones en forma sólida que lo que se pierde por evaporación durante un año hidrológico.

El proceso finaliza mediante la formación de lagunas o fusión rápida de los mantos de nieve (figura 09), que acelera la pérdida de extensas zonas de nieve (estratos de nieve fresca y estratos de nieve de acumulación de 2 y 3 meses). Al fluir los volúmenes de agua que se desplaza por las grietas, lubrican la base del hielo provocando deslizamientos acelerados del glaciar. Este proceso se está observando en la zona de ablación conocido como lengua del glaciar, en donde se ha incrementado la formación de lagunas y grietas por donde fluye el agua (figura 10). Estas formaciones tienen áreas considerables y además, constituye una amenaza para los mismos turistas que visitan el nevado Huaytapallana.

La zonas altas comprendidas entre los 5050 y 5200 msnm, carecen de estos impactos antropogénicos (figura 11). La elevación y las condiciones de montaña agreste no permiten que se desarrolle actividades de turismo. La formación de hielo por procesos de diagénesis es el más visible a estas altitudes. Estos mecanismos como lo menciona Francou (4) son muestras del impacto directo de los seres humanos.

La reforestación constituye una actividad indirecta de impacto sobre los glaciares, a diferencia de los glaciares alpinos que se ubican en las latitudes altas, donde los bosques han constituido un mecanismo natural dentro del proceso de formación de grandes eras glaciares. El implantar y modificar la cobertura y fisiografía de las zonas aledañas al glaciar modifican la isoterma. El punto de congelación de cero grados centígrados se eleva con estas actividades, acercándose a las altitudes cercanas al glaciar, como lo menciona Maisincho (3).

El pastoreo, constituye la tercera actividad que tiene un impacto indirecto a los glaciares, especialmente en las zonas de ablación, al acelerar la pérdida de glaciares por estas actividades que modifican el paisaje y destruyen los pastos naturales que por años constituían la forma natural de soporte de acumulación de agua como lo menciona Kaser (10).

Las zanjas de infiltración y las actividades culturales de costumbres de la población se posicionan en el último eslabón de los efectos indirectos, porque los impactos aún 
son menores, debido a que estas actividades se realizan en zonas alejadas de los glaciares.

\section{Agradecimientos}

A Wilfredo Bulege, José Barrios y Esau Caro de la Universidad Continental; al Instituto Geofísico del Perú; y a Edsel Arroyo, Wyler Espeza y Frans Cornejo.

\section{REFERENCIAS BIBLIOGRÁFICAS}

1. Arroyo AJ. Cambios en el régimen hídrico de la laguna Lasuntay y Chuspicocha por variaciones en el nevado Huaytapallana. Apuntes de Ciencia \& Sociedad. 2011 ; 01 (02): 141 150.

2. Arroyo AJ. Tendencias y cambio del clima del Valle del Mantaro mediante los análisis de índices de precipitación efectiva y temperatura eficiente. Apuntes de Ciencia \& Sociedad". 2011; $01(01): 45-54$.

3. Maisincho J, Mendoza J, Ramírez A, Soruco J, Taupin D, Wagnon P. Métodos de Observación de Glaciares en los Andes Tropicales, Mediciones de Terreno y Procesamiento de Datos. París: Ediciones IRD; 2004.

4. Francou B, Ribstein P, Wagnon P, Ramirez E, Pouyaud B. Glaciers of the tropical Andes: indicators of global climate variability. In: Huber, U., Bugmann, H.K.M., Reasoner, M.A. (Eds.), Global Change and Mountain Regions: An Overview of Current Knowledge, vol. 23. Springer, Dordrecht: Ediciones IRD; 2005.

5. Brasseur G. Physique et Chemique de l'Atmosphère moyenne, Masson. El Fin de las Cumbres Nevadas, Glaciares y Cambio Climático en la comunidad Andina. Paris: Ediciones Española de Cooperación Internacional; 2007.

6. Hussein A, et al. GEO 5. Perspectivas del Medio Ambiente Mundial. Programa de las Naciones Unidas para el Medio Ambiente PNUMA. Nairobi: UNEP; 2012.

7. Baird D. An Introduction to Measurement Theory and Experiment Desig. HJ: 3rd. ed. Prentice Hall, Englewood Cliffs; 1995.

8. Dyurgerov M. Glacier Mass Balance and Regime: Data of Measurements and Analysis. Occasional Paper No. 55, University of Colorado, Boulder; 2002.

9. Francou B, Ribstein P, Saravia R. Monthy Balance and Water discharge on an intertropical glacier. Bolivia: $16^{\circ} \mathrm{S}$. Journal of Glaciology; 1995.

10. Francou B, Vuille M, Wagnon P, Mendoza J, Sicart JE. Tropical Cimate Change Recorded by a Glacier of the Central Andes During the Last Decades of the 20th century. Chacaltaya: Journal of Geophysical Research; 2003.

11. Kaser G, Osmaston H. Tropical Glaciers. Cambridge: University Press - United Kingdom; 2002.

12. Oerlemans J, Fortuin J. Sensitivity of glaciers and small ice caps to Greenhouse warming, Berlin; 2002.

13. Ribstein P, Tiriau E, Francou B, Saravia F. Tropical climate and glacier hydrology: A case study in Bolivia, Journal of Hydrology; 1995.

14. Pouyaud B, Francou P, Ribstein P. Un réseau d'observation des glaciers dans les Andes tropicales, Bulletin de l'institut français d'études andines; 1995.

15. Dyugerov J, Paterson V. Analysis of water of glaciers and snowfall caps to Greenhouse warming. Londres: 2006.

Correo electrónico:

jarroyox@gmail.com 